\title{
Medicinal plants of Otwal and Ngai Sub Counties in Oyam District, Northern Uganda
}

Maud M Kamatenesi ${ }^{1 \dagger}$, Annabel Acipa $^{2^{*}}$, Hannington Oryem-Origa ${ }^{1 \dagger}$

\begin{abstract}
Background: An ethnobotanical study was carried out in four parishes in the Ngai and Otwal Sub Counties in Oyam district, Northern Uganda, where insurgency has been prevalent for the past 20 years. Documenting medicinal plant species used in treating various health conditions among the local people.

Methods: Information was obtained from mainly the local population, the traditional healers and other experienced persons through interviews, formal and informal discussions and field excursions.

Results: Seventy one plant species were reported for use in the treatment of various diseases in the study area. These plant species belongs to 41 families, with Asteraceae being the most represented. Roots were ranked the commonest plant part used. Oral administration was the most frequently used route of administration. A total of 41 different health conditions were reported to be treated by use of medicinal plant species. Thirty nine percent of the recorded plant species were reported for treating stomach related ailments.

Conclusion: The use of medicinal plants in primary healthcare is still a common practice in Ngai and Otwal Sub Counties. The trust they have is built on the curative outcome properties claimed, poverty and armed conflict that lead to inadequate healthcare facilities. The generation gap caused by the over 20 years of insurgency in the area has brought about knowledge gap on the usage of medicinal plant species between the young and the older generation.
\end{abstract}

\section{Background}

World wide over $80 \%$ of the people depend on medicinal plant species to meet their day today healthcare needs [1]. Rural household of Uganda rely heavily on plant resources for food, fodder and herbal medicine [2]. Tabuti [2] further asserted that savanna environment contains many plant resources of economic values such as foods and medicines. These resources are widely relied on by rural communities in developing countries because of inefficiencies in service delivery or because social services and goods are unaffordable. For this reason many people are currently resorting to traditional medicine for primary health care due to high costs in accessibility, cultural compatibility, self-reliance among others [3]. They also employ herbal medicines because of cultural preferences and perceived effectiveness $[4,5]$.

\footnotetext{
* Correspondence: aamiraclenet@yahoo.co.uk

† Contributed equally

${ }^{2}$ Institute of Environment and Natural Resources Makerere University, P.O Box 7062, Kampala, Uganda

Full list of author information is available at the end of the article
}

Medicinal plant species form a main part of treatment for the rural poor. Traditional medicine usage in rural Ugandan population for day-to-day health care needs is close to 90\% [6]. Kamatenesi and Oryem [6] further reported that women and children form the bulk of the people reliant on herbal medicine. According to Katuura et al [7], malaria was reported to be the most common condition treated by traditional healers in Mbarara District. The use of traditional herbal remedies is encountered in both rural and urban areas in Mali and that traditional medicine is one of the surest means to achieve total health care coverage for African's population [8].

Discourses on the future of traditional medicine in Africa and other indigenous societies often assume government recognition and integration into the formal health care systems [9].

In certain areas in Nigeria, the only health care providers close to the people are the traditional medical practitioners [10]. However, it should be noted that medicinal plant species have also been discovered to have other

\section{() Biomed Central}


uses as some could be used as vegetables, fruits, trees and ornamentals [11].

Health services in Oyam District are inadequate, and only 15 out of the 43 parishes in Oyam District have health facilities. Maternal mortality rate is still high because clean and safe deliveries are at only 14\% because it is mainly the traditional birth attendants (TBA) who play a significant role [12].

\section{Methods}

\section{Study area}

This study was carried out in Ngai and Otwal sub counties in Oyam District which is situated in northern Uganda on coordinates $02^{\circ} 14^{\prime} \mathrm{N} 32^{\circ} 23^{\prime} \mathrm{E}$ (Figure 1) [13]. The sampling sites were located in the Parishes of Aramita, Akuca and Omac from Ngai Sub County and Abela from Otwal Sub-County. The study was conducted between August 2007 and February 2008 in Oyam District, Northern Uganda.

\section{Data collection}

Ethnobotanical information was obtained through informed consent semi-structured interviews with key informants. The key informants consisted of health workers, renowned herbalists, and local leaders. However, the bulk of the respondents were local residents who were identified through household numbers. Knowledge on the use of medicinal plant species was documented, the local name of plant species, diseases or ailments they treat, part of plant used, methods of preparation and administration were recorded.

In addition, a total of 84 households were interviewed using questionnaires, after being randomly chosen from the total household list from the LC I (Local Councilor One) chairperson. Forty four households from Ngai and another 40 from Otwal Sub Counties were interviewed through the use of questionnaire. Some questions asked included; village of respondent, level of education, knowledge on medicinal plant species among others.

For more studies and information, three focus group discussions were conducted in Acandano village in Ngai Sub-County and Abela primary school and Ojwi centre in Otwal Sub-County. In this case the respondents were asked research guided questions. The groups comprised of children 15, women 20 and men 12. The groups participated voluntarily at the invitation of LC 1 chairman. The focus group discussion helped discover the extent of distribution of knowledge on medicinal plant species.

\section{Voucher Specimens and Sample Collection}

Voucher specimens of the documented plant species were collected according to standard practice, including roots, flowers, and fruits where possible [14]. Collection only involved samples that were identified by the respondent. The voucher specimens were delivered to Makerere University Botany Herbarium where further identification and classification was done. Scientific names of plant species were identified based on International Plant Name Index (IPNI: http://www.ipni.org).

\section{Results}

A total of 110 respondents were interviewed from the study area; 46 were females and males were 64 as shown in table 1.

From the research findings, 71 medicinal plant species both wild and cultivated belonging to 42 families were documented and identified in the study area (Table 2). The family Asteraceae (5 species) was the most represented followed by Leguminosae and Lamiaceae (4 species) plant species each; Solanaceae, Poaceae, Eurphorbiaceae, and Zingiberaceae had 3 plant species in each family, and the remaining families had two and one species. With regard to growth habits, the plant species consisted of shrubs (39\%), herbs and climbers (36.6\%), trees (21\%) and grasses (4\%).

These plant species were mainly obtained from open grassland area (41\%), garden or farms $(21 \%)$, homestead (13\%) wooded grassland $11 \%$, forest (7\%) and least number was obtained from swamps (4\%) and forest edge (3\%).

The most commonly mentioned plant species by respondents were Clerodendrum umbellatum Poir (25\%) Securidaca longipedunculata Fres. (17\%) while the least mentioned among respondents includes; Crotalaria ochroleuca G.Don, Albizia coriaria Welw (0.9\%). Fifty five percent of the plant species mentioned were used to treat more than one disease and $45 \%$ to treat only one disease.

A total of plant species documented, $25 \%$ were edible and formed part of local diet (Table 2). Fifty five percent of these were used in the treatment of more than one disease while $45 \%$ were believed to treat only one particular disease. The conservation status of the medicinal plant species is such that only $10 \%$ were cultivated and $90 \%$ were collected from the wild (Table 2).

Roots were the commonest plant parts $(57 \%)$ being used; followed by leaves (23\%) (Figure 2). The most underutilized plant part were found to be flowers with only $2 \%$ usage, fruits making up $3 \%$ and the rest of plant parts harvested making up $4 \%, 5 \%$ and $7 \%$ of stems, seeds and bark respectively.

Records reveal that a total of 41 conditions were treated with medicinal plant parts in Otwal and Ngai subcounties in Oyam District. The common condition being treated in Ngai and Otwal sub counties was found to be abdominal pains and this was reported by $11 \%$ of the respondents, followed by cough at $10 \%$. Other conditions such as wounds had 5.6\% headache; epilepsy and STD/STI at $4.6 \%$. Those least mentioned at below $1 \%$ were impotence, toothache, cholera, fever among others. 


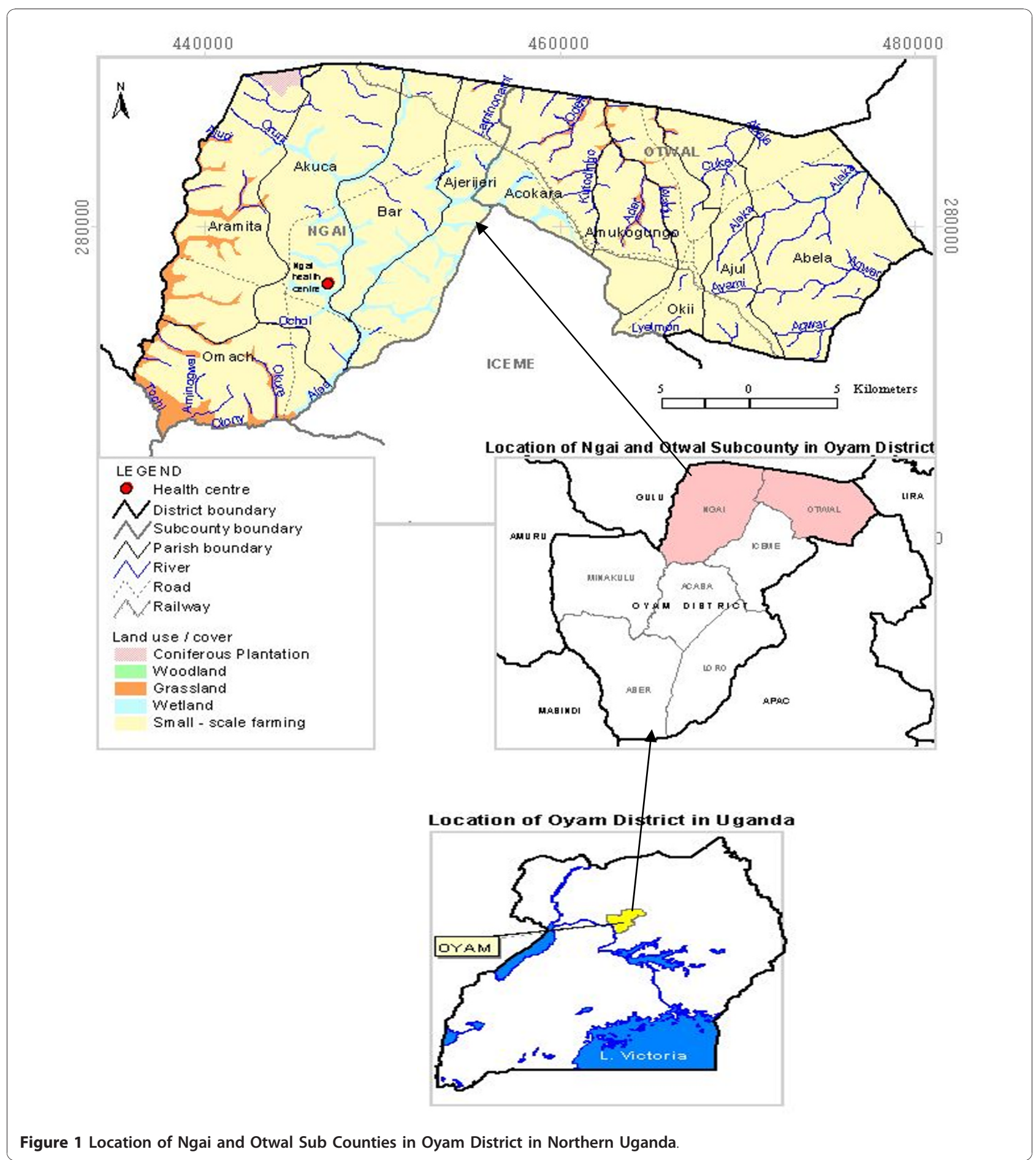

The most common way of preparing these medicinal plant species was mainly by crushing and extracting using cold water making up an overall $48 \%$. This was followed by crushing plant parts and applied in that form at $20 \%$. The least mode was found to be burning, and adding the ashes into bath water making up less that $1 \%$.
On administration, oral administration through drinking was found to be the most frequently used at $69 \%$ and the least were through bathing with, massaging and smoking at less than $1 \%$.

The main sources of indigenous knowledge of medicinal plant species were parents at $40 \%$, grandparents at $35 \%$ (Table 3 ). The least sources of information about 


\begin{tabular}{|c|c|c|c|}
\hline \multicolumn{2}{|l|}{ Respondents } & \multicolumn{2}{|r|}{ Total } \\
\hline Males & Females & & \\
\hline $64(58 \%)$ & $46(42 \%)$ & & 110 \\
\hline \multicolumn{4}{|c|}{ Age Characteristics of Respondents } \\
\hline 13-24 years & 25-37 years & $38-49$ years & 50 years and above \\
\hline 17 (15\%) & 32 (29\%) & 27 (25\%) & 34 (31\%) \\
\hline
\end{tabular}

medicinal plant species were through dreams at $3.8 \%$ and in-laws $2.9 \%$.

The use of medicinal plant species was found to be driven mainly by its perceived effectiveness (34\%), poverty, medical facilities being far $(23 \%)$ and lack of medicines in hospitals (5\%) (Table 4). The least use of medicinal plant species was due to referral from medical personnel (3\%).

\section{Discussion}

The 71 medicinal plant species of cultivated and wild types were greatly utilized by people of Oyam District as herbal remedies. These plant species fall under 42 families, with the family Asteraceae having the highest number of medicinal plant species. The family Asteraceae was also recorded as having the most number of medicinal plant species as other studies in other areas also reveals $[15,16]$. Clerodendrum umbellatum, Securidaca longipedunculata, Clematis hirsuta and Conyza sumaternsis were among the most frequently utilized species. The frequency of mention of a given plant species could be an indication of the prevalence of a given condition it can treat and its therapeutic values.

Roots were the most commonly harvested plant part of the medicinal plants compared to any other part. This form of harvesting however, is threatening to the survival of the plant. Plant species such as Lantana camara, Urtica massaica had leaves and roots being harvested. Harvesting of two or more plant parts can be more damaging especially when the roots and barks/ stem are harvested. Thus from the conservation point of view, the high utilization of roots of plant species in Oyam District put these plant species at a risk because of the damages inflicted on the plant species. This was also noted in other areas [6].

Many of these plant species treated more than one condition and are being used in combination. This pattern of using medicinal plant species for varying conditions was also observed among the local communities in Mabira Forest Reserve area [15]. However, it was found that locals usually mix the medicinal plant species to ensure effectiveness in treating a given ailment $[17,18]$. This was also observed in Ngai Sub County, where the extent of knowledge of medicinal plant mixing determined the success of a traditional healer. Medicinal plant are strongly believed by the local people of Ngai and Otwal to be effective and this among other reasons explain why they have continued to use them, thus their reliance on them for basic healthcare. This trend was also observed among the people living around Queen Elizabeth National Park in western Uganda [3,6]

Abdominal pain and cough were the most frequently treated ailments. These are diseases associated with personal hygiene. The study area has had IDP camps which was always associated with poor hygiene and overcrowding. The high frequency of mention of these diseases were directly associated with the high prevalence of these diseases in the area. This goes on to explain why many of the medicinal plant species mentioned were used for treating these ailments indicating widespread knowledge of medicinal plant species used for their treatment. For example, 25\% of respondents mentioned that Clerodendrum umbellatum was used for treatment of abdominal pain.

The most common method of preparation of medicinal plant species before being administered was found to be applied to most plant species. This involved crushing and extracting plant materials using cold/warm water and boiling. Those that were boiled were effectively extracted compared to use of cold water, since boiling also preserves the medicine longer. Oral administration was noted as number one mode of dispensing of herbal medicine. This mode of administration of herbal medicine was also reported elsewhere $[6,18]$.

Some of these plant species are popular and used all over Uganda and are on sale in most markets. For instance Cleome gynandra, Cajanus cajan, Vitallaria paradoxum, Capsicum frutescens were found to be sources of food and were being eaten not only locally but also nationally and internationally [3].

Some studies carried out in and outside Uganda showed that some of these plant species were potent as medicine. A plant like Aspilia africana, is said to have high antiplasmodial activity [19]. Some other plant species mentioned elsewhere as medicine include Cassia occidentalis which is used in Burkina Faso as stimulant [3].

\section{Conservation issues}

It should be noted that a high percentage of these plant species are harvested from the wild, but with no consideration for domestication hence threatening their existence. The plant species are being overexploited, and the rapid environmental degradation coupled with insurgency has put mounting pressure on the environment. This may lead to the disappearance of many species of medicinal plants of economic value. According to one of the local traditional practitioners, Okello Okiko, the use of medicinal plant species is becoming expensive since 
Table 2 Medicinal plants their habits, growth habit, frequency of mention, plant part used, diseases treated, methods of preparation and administration

\begin{tabular}{|c|c|c|c|c|c|c|c|c|c|}
\hline Family & Taxon & Habitat & Habit & $\begin{array}{l}\text { Plant } \\
\text { part } \\
\text { used }\end{array}$ & Disease & $\begin{array}{l}\text { Number } \\
\text { of } \\
\text { diseases } \\
\text { treated }\end{array}$ & $\begin{array}{l}\text { Freq of } \\
\text { mention } \\
\text { of plant }\end{array}$ & $\begin{array}{l}\text { Methods of } \\
\text { Preparation }\end{array}$ & Administration \\
\hline Amaranthaceae & $\begin{array}{l}\text { Pupalia lappacea } \\
\text { Juss. AA-49-07 }\end{array}$ & $\begin{array}{l}\text { Wooded } \\
\text { grassland }\end{array}$ & $\mathrm{SH}$ & R & Syphilis & 1 & 2 & $\begin{array}{l}\text { Crushed, } \\
\text { boiled* }\end{array}$ & Extract drunk \\
\hline \multirow[t]{2}{*}{ Anacardiaceae } & $\begin{array}{l}\text { Mangifera indica } \\
\text { L. AA-53-07 }\end{array}$ & Homestead & $\mathrm{T}$ & B & Diarrhoea & 2 & 8 & $\begin{array}{l}\text { Crushed, } \\
\text { mixed in cold } \\
\text { water }\end{array}$ & Extract drunk twice a day \\
\hline & & & & R & Cough & & & $\begin{array}{l}\text { Crushed, } \\
\text { mixed in cold } \\
\text { water }\end{array}$ & Extract drunk \\
\hline \multirow[t]{2}{*}{ Apocynaceae } & $\begin{array}{l}\text { Carissa edulis } \\
\text { (Forssk) Vahl. } \\
\text { AA-59-07 }\end{array}$ & Grassland & $\mathrm{SH}$ & R & Epilepsy & 2 & 3 & $\begin{array}{l}\text { Crushed, } \\
\text { mixed in cold } \\
\text { water }\end{array}$ & Extract drink \\
\hline & & & & S & $\begin{array}{l}\text { Abdominal } \\
\text { pain }\end{array}$ & & & $\begin{array}{l}\text { Crushed, } \\
\text { mixed in cold } \\
\text { water }\end{array}$ & Extract drunk \\
\hline \multirow[t]{3}{*}{ Asclepiadaceae } & $\begin{array}{l}\text { Mondia whiteii } \\
\text { Skeels AA-57-07 }\end{array}$ & Forest & C & $\mathrm{R}$ & Flu, cold & 4 & 8 & $\begin{array}{l}\text { Crushed, } \\
\text { mixed in cold } \\
\text { water }\end{array}$ & Extract drunk \\
\hline & & & & $R$ & $\begin{array}{l}\text { Abdominal } \\
\text { pain }\end{array}$ & & & $\begin{array}{l}\text { Crushed, } \\
\text { mixed in cold } \\
\text { water }\end{array}$ & Extract drunk twice a day \\
\hline & & & & $R$ & $\begin{array}{l}\text { Headache, } \\
\text { cough }\end{array}$ & & & $\begin{array}{l}\text { Picked, } \\
\text { cleaned }\end{array}$ & Chewed \\
\hline Asparagaceae & $\begin{array}{l}\text { Asparagus } \\
\text { africanus Hochst. } \\
\text { ex.A. Rich } \\
\text { AA-48-07 }\end{array}$ & $\begin{array}{l}\text { Open } \\
\text { grassland }\end{array}$ & $\mathrm{SH}$ & $\mathrm{R}$ & $\begin{array}{l}\text { Swollen } \\
\text { body }\end{array}$ & 1 & 4 & $\begin{array}{l}\text { Crushed, } \\
\text { mixed in cold } \\
\text { water }\end{array}$ & $\begin{array}{l}\text { Extract drunk one glass } \\
\text { twice a day, rub on skin } \\
\text { cuts }\end{array}$ \\
\hline \multirow[t]{9}{*}{ Asteraceae } & $\begin{array}{l}\text { Acmela canlirhiza } \\
\text { Delile AA-64-07 }\end{array}$ & $\begin{array}{l}\text { Garden } \\
\text { edge, road } \\
\text { side }\end{array}$ & $\mathrm{H}$ & $R, L$ & Cough & 2 & 2 & $\begin{array}{l}\text { Dried, } \\
\text { powdered }\end{array}$ & $\begin{array}{l}\text { Extract drunk three } \\
\text { teaspoon twice a day }\end{array}$ \\
\hline & & & & $\mathrm{R}$ & $\begin{array}{l}\text { Retained } \\
\text { placenta }\end{array}$ & & & $\begin{array}{l}\text { Crushed, } \\
\text { mixed in } \\
\text { warm water* }\end{array}$ & Extract drunk \\
\hline & $\begin{array}{l}\text { Biden pilosa L. } \\
\text { AA-47-07 }\end{array}$ & Garden & $\mathrm{H}$ & L & Wounds & 1 & 3 & $\begin{array}{l}\text { Dried, } \\
\text { powdered }\end{array}$ & Applied on wound \\
\hline & $\begin{array}{l}\text { Echinops } \\
\text { amplexicaulis Oliv. } \\
\text { AA-07-07 }\end{array}$ & $\begin{array}{l}\text { Open } \\
\text { grassland }\end{array}$ & $\mathrm{SH}$ & R & Hydrocelle & 7 & 7 & $\begin{array}{l}\text { Crushed, } \\
\text { mixed in cold } \\
\text { water }\end{array}$ & $\begin{array}{l}\text { Extract drunk three times } \\
\text { a day }\end{array}$ \\
\hline & & & & $\mathrm{R}$ & $\begin{array}{l}\text { Hernia } \\
\text { scrotal }\end{array}$ & & & $\begin{array}{l}\text { Crushed, } \\
\text { mixed in cold } \\
\text { water* }\end{array}$ & Extract drunk \\
\hline & & & & R & Stomachache & & & $\begin{array}{l}\text { Crushed, } \\
\text { boiled }\end{array}$ & $\begin{array}{l}\text { Extract drunk } 200 \mathrm{ml} \\
\text { once a day }\end{array}$ \\
\hline & & & & $R$ & TB & & & $\begin{array}{l}\text { Crushed, } \\
\text { boiled }\end{array}$ & $\begin{array}{l}\text { Extract drunk quarter } \\
\text { glass for adults twice a } \\
\text { day, two spoonful twice } \\
\text { a day for children }\end{array}$ \\
\hline & & & & $\mathrm{R}$ & $\begin{array}{l}\text { snake bite, } \\
\text { whooping } \\
\text { cough, } \\
\text { syphilis }\end{array}$ & & & $\begin{array}{l}\text { Crushed, } \\
\text { mixed in cold } \\
\text { water }\end{array}$ & $\begin{array}{l}\text { Extract drunk one glass } \\
\text { twice a day }\end{array}$ \\
\hline & $\begin{array}{l}\text { Conyza } \\
\text { sumatrensis } \\
\text { (Retz.) E.Walker } \\
\text { AA-35-07 }\end{array}$ & $\begin{array}{l}\text { Open } \\
\text { grassland }\end{array}$ & $\mathrm{SH}$ & L & Wounds & 3 & 12 & Crushed & Juice onto fresh wound \\
\hline
\end{tabular}


Table 2 Medicinal plants their habits, growth habit, frequency of mention, plant part used, diseases treated, methods of preparation and administration (Continued)

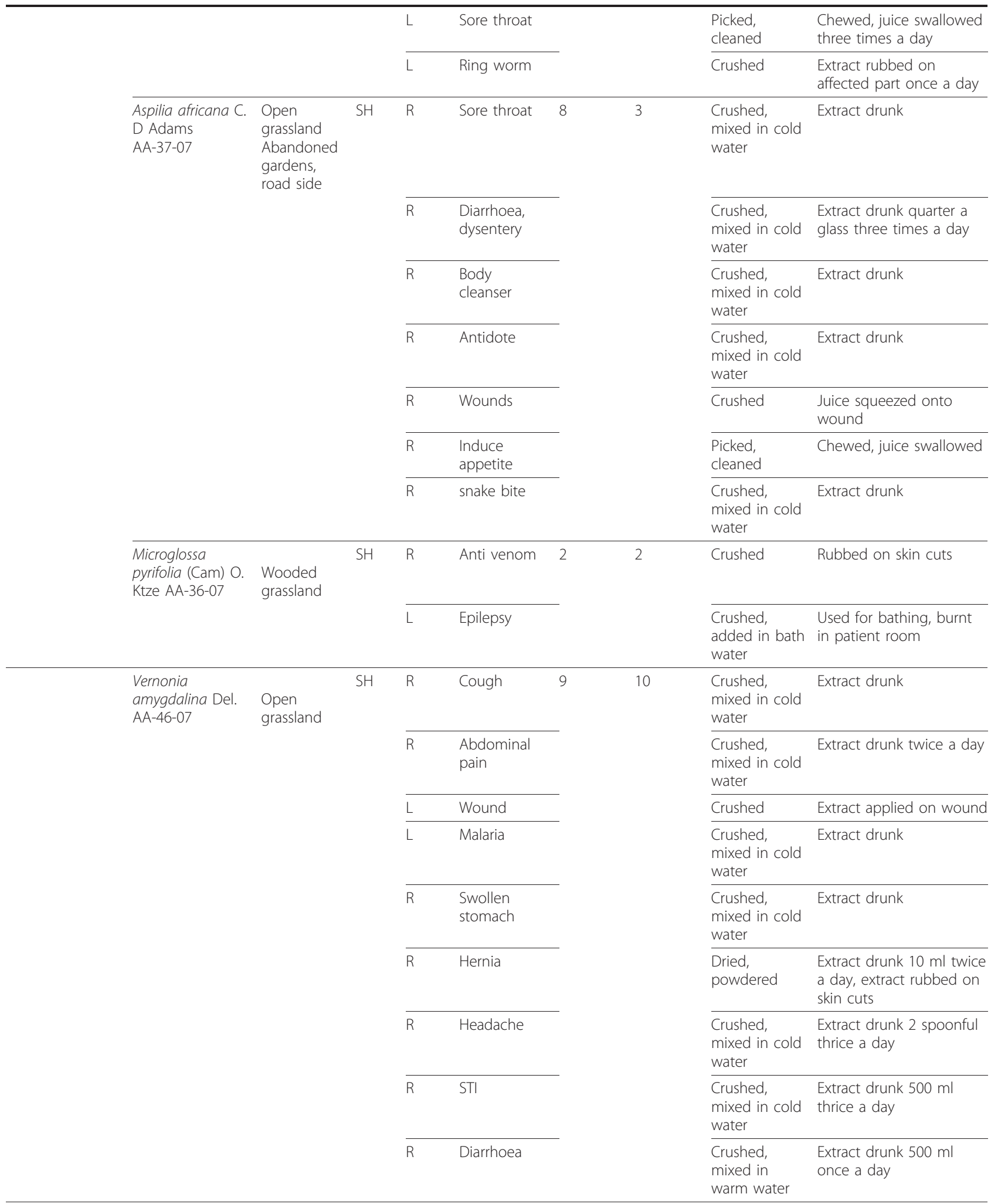


Table 2 Medicinal plants their habits, growth habit, frequency of mention, plant part used, diseases treated, methods of preparation and administration (Continued)

\begin{tabular}{|c|c|c|c|c|c|c|c|c|c|}
\hline & $\begin{array}{l}\text { Vernonia sp. } \\
\text { AA-02-07 }\end{array}$ & $\begin{array}{l}\text { Open } \\
\text { grassland, } \\
\text { garden }\end{array}$ & $\mathrm{H}$ & $R, L$ & $\begin{array}{l}\text { Backbone } \\
\text { disease }\end{array}$ & 1 & 3 & $\begin{array}{l}\text { Crushed, } \\
\text { boiled, } \\
\text { Heated over } \\
\text { fire }\end{array}$ & $\begin{array}{l}\text { Extract drunk, heated } \\
\text { leaves massage body } \\
\text { twice a day }\end{array}$ \\
\hline \multirow[t]{5}{*}{ Bignoniaceae } & $\begin{array}{l}\text { Markhamia } \\
\text { platycalyx } \\
\text { Sprague } \\
\text { AA-54-07 }\end{array}$ & $\begin{array}{l}\text { Wooded } \\
\text { grassland }\end{array}$ & $\mathrm{T}$ & R & $\begin{array}{l}\text { Ease child } \\
\text { bearing, } \\
\text { Induces } \\
\text { labour }\end{array}$ & 1 & 1 & $\begin{array}{l}\text { Crushed, } \\
\text { mixed in } \\
\text { warm water }\end{array}$ & $\begin{array}{l}\text { Extract drunk one glass } \\
\text { once a day }\end{array}$ \\
\hline & $\begin{array}{l}\text { Stereospermum } \\
\text { kunthianum } \\
\text { Cham. AA-55-07 }\end{array}$ & $\begin{array}{l}\text { Wooded } \\
\text { grassland }\end{array}$ & $\mathrm{T}$ & R & Wounds & 1 & 1 & $\begin{array}{l}\text { Dried, } \\
\text { powdered }\end{array}$ & Applied on wound \\
\hline & $\begin{array}{l}\text { Kigelia africana } \\
\text { (Lam.) Benth } \\
\text { AA-60-07 }\end{array}$ & $\begin{array}{l}\text { Wooded } \\
\text { grassland }\end{array}$ & T & L & Eye disease & 3 & 2 & Crushed & Squeezed in eye \\
\hline & & & & B & $\begin{array}{l}\text { Poison } \\
\text { antidote }\end{array}$ & & & $\begin{array}{l}\text { Crushed, } \\
\text { boiled }\end{array}$ & Extract drunk once a day \\
\hline & & & & S & Impotence & & & $\begin{array}{l}\text { Dried, } \\
\text { powdered }\end{array}$ & Extract drunk, eaten. \\
\hline \multirow[t]{2}{*}{ Caesalpiniaceae } & $\begin{array}{l}\text { Cassia siamea } \\
\text { Lam. AA-56-07 }\end{array}$ & $\begin{array}{l}\text { Semi } \\
\text { cultivated }\end{array}$ & $\mathrm{T}$ & R & Sore throat & 2 & 4 & $\begin{array}{l}\text { Crushed and } \\
\text { mixed in cold } \\
\text { water }\end{array}$ & Extract drunk \\
\hline & & & & L & $\begin{array}{l}\text { Abdominal } \\
\text { pain }\end{array}$ & & & $\begin{array}{l}\text { Picked, } \\
\text { cleaned }\end{array}$ & $\begin{array}{l}\text { Chewed, liquid } \\
\text { swallowed }\end{array}$ \\
\hline \multirow[t]{3}{*}{ Capparaceae } & $\begin{array}{l}\text { Cleome gynandra } \\
\text { L.AA-61-07 }\end{array}$ & $\begin{array}{l}\text { Homestead, } \\
\text { garden }\end{array}$ & $\mathrm{H}$ & L & Headache & 3 & 5 & Crushed & Rubbed on forehead \\
\hline & & & & $\mathrm{L}$ & Ring worm & & & Crushed & Rubbed on affected area \\
\hline & & & & $R$ & Eye disease & & & Crushed & Dropped in eye \\
\hline Caricaceae & $\begin{array}{l}\text { Carica papaya L. } \\
\text { AA- } 43-07\end{array}$ & Homestead & $\mathrm{T}$ & $\mathrm{R}$ & $\begin{array}{l}\text { body pain by } \\
\text { witch craft }\end{array}$ & 1 & 3 & Crushed & $\begin{array}{l}\text { Rubbed on body twice a } \\
\text { day }\end{array}$ \\
\hline \multirow[t]{2}{*}{ Celastraceae } & $\begin{array}{l}\text { Maytenus } \\
\text { senegalensis } \\
\text { (Lam) Exell } \\
\text { AA-45-07 }\end{array}$ & Forest & T & R & Epilepsy & 2 & 1 & $\begin{array}{l}\text { Crushed, } \\
\text { mixed in cold } \\
\text { water }^{*}\end{array}$ & $\begin{array}{l}\text { Extract drunk } 50 \mathrm{ml} \text { three } \\
\text { times a day }\end{array}$ \\
\hline & & & & $\mathrm{R}$ & Miscarriage & & & $\begin{array}{l}\text { Crushed, } \\
\text { mixed in cold } \\
\text { water }\end{array}$ & $\begin{array}{l}\text { Extract drunk } 300 \mathrm{ml} \text { two } \\
\text { times a day }\end{array}$ \\
\hline \multirow[t]{2}{*}{ Chenopodiaceae } & $\begin{array}{l}\text { Chenopodium } \\
\text { ambrosioides L. } \\
\text { AA-50-07 }\end{array}$ & $\begin{array}{l}\text { Around } \\
\text { home stead }\end{array}$ & $\mathrm{H}$ & $L$ & Headache & 2 & 4 & $\begin{array}{l}\text { Crushed, } \\
\text { mixed in hot } \\
\text { water }\end{array}$ & $\begin{array}{l}\text { Steam inhaled, heated } \\
\text { leaves placed on face }\end{array}$ \\
\hline & & & & L & Epilepsy & & & $\begin{array}{l}\text { Crushed, } \\
\text { mixed in cold } \\
\text { water }\end{array}$ & $\begin{array}{l}\text { Extract drunk } 25 \mathrm{ml} \text { twice } \\
\text { a day, applied on skin } \\
\text { cuts }\end{array}$ \\
\hline \multirow[t]{5}{*}{ Combretaceae } & $\begin{array}{l}\text { Combretum molle } \\
\text { R.Br.G. Don } \\
\text { AA-44-07 }\end{array}$ & $\begin{array}{l}\text { Swampy } \\
\text { area, forest } \\
\text { edge }\end{array}$ & $\mathrm{T}$ & R & Cough & 1 & 1 & $\begin{array}{l}\text { Dried, } \\
\text { powdered } \\
\text { added into } \\
\text { one glass of } \\
\text { water }\end{array}$ & Drunk twice a day \\
\hline & $\begin{array}{l}\text { Combretum } \\
\text { collinum Fresen } \\
\text { AA-42-07 }\end{array}$ & $\begin{array}{l}\text { Open } \\
\text { grassland }\end{array}$ & $\mathrm{T}$ & L & Cough & 4 & 12 & $\begin{array}{l}\text { Crushed, } \\
\text { mixed in cold } \\
\text { water* }\end{array}$ & Extract drunk twice a day \\
\hline & & & & $R, B$ & Wounds & & & Crushed & $\begin{array}{l}\text { Juice squeezed on } \\
\text { wound }\end{array}$ \\
\hline & & & & $R, B$ & Diarrhoea, & & & $\begin{array}{l}\text { Crushed, } \\
\text { mixed in cold } \\
\text { water }\end{array}$ & $\begin{array}{l}\text { Extract drunk } 4 \text { teaspoon } \\
\text { twice a day }\end{array}$ \\
\hline & & & & $R, B$ & $\begin{array}{l}\text { Abdominal } \\
\text { pain }\end{array}$ & & & $\begin{array}{l}\text { Crushed, } \\
\text { mixed in cold } \\
\text { water }\end{array}$ & \\
\hline
\end{tabular}


Table 2 Medicinal plants their habits, growth habit, frequency of mention, plant part used, diseases treated, methods of preparation and administration (Continued)

\begin{tabular}{|c|c|c|c|c|c|c|c|c|c|}
\hline \multirow[t]{4}{*}{ Cucurbitaceae } & $\begin{array}{l}\text { Cucurbita } \\
\text { maxima Wall. } \\
\text { AA-38-07 }\end{array}$ & $\begin{array}{l}\text { Gardens, } \\
\text { antihill }\end{array}$ & C & R & $\begin{array}{l}\text { Abdominal } \\
\text { pain }\end{array}$ & 1 & 1 & $\begin{array}{l}\text { Crushed, } \\
\text { mixed in cold } \\
\text { water }\end{array}$ & Extract drunk \\
\hline & $\begin{array}{l}\text { Momordica } \\
\text { foetida Schum. } \\
\text { AA-52-07 }\end{array}$ & Antihill & C & R & STI & 3 & 2 & $\begin{array}{l}\text { Crushed, } \\
\text { mixed in cold } \\
\text { water }\end{array}$ & $\begin{array}{l}\text { Extract drunk one glass } \\
\text { once a day }\end{array}$ \\
\hline & & & & $R$ & $\begin{array}{l}\text { Cough, } \\
\text { abdominal } \\
\text { pain }\end{array}$ & & & $\begin{array}{l}\text { Crushed, } \\
\text { mixed in cold } \\
\text { water }\end{array}$ & $\begin{array}{l}\text { Extract drunk one glass } \\
\text { twice a day }\end{array}$ \\
\hline & $\begin{array}{l}\text { Kedrostis } \\
\text { foetidissima Cogn. } \\
\text { AA-41-07 }\end{array}$ & $\begin{array}{l}\text { Open } \\
\text { grassland }\end{array}$ & C & R & Measles & 1 & 1 & $\begin{array}{l}\text { Crushed, } \\
\text { mixed in cold } \\
\text { water }\end{array}$ & Extract drunk once a day \\
\hline Dioscoreaceae & $\begin{array}{l}\text { Dioscorea sp } \\
\text { AA-62-07 }\end{array}$ & Garden & $\mathrm{H}$ & L & $\begin{array}{l}\text { Loss of } \\
\text { appetite }\end{array}$ & 1 & 1 & $\begin{array}{l}\text { Crushed, } \\
\text { boiled }\end{array}$ & Eaten \\
\hline \multirow[t]{3}{*}{ Eurphorbiaceae } & $\begin{array}{l}\text { Euphorbia hirta L. } \\
\text { AA-71-07 }\end{array}$ & $\begin{array}{l}\text { Garden, } \\
\text { along } \\
\text { roadside }\end{array}$ & $\mathrm{H}$ & R & Cough & 2 & 6 & $\begin{array}{l}\text { Crushed, } \\
\text { mixed in cold } \\
\text { water }\end{array}$ & $\begin{array}{l}\text { Extract drunk three times } \\
\text { a day }\end{array}$ \\
\hline & & & & St & Fresh wound & & & Sap collected & $\begin{array}{l}\text { Applied on wound two } \\
\text { times a day }\end{array}$ \\
\hline & $\begin{array}{l}\text { Fluggae virosa } \\
\text { (Willd.) Voigt } \\
\text { AA-40-07 }\end{array}$ & $\begin{array}{l}\text { Wooded } \\
\text { grassland }\end{array}$ & $\mathrm{SH}$ & R & Miscarriage & 1 & 2 & $\begin{array}{l}\text { Crushed, } \\
\text { mixed in cold } \\
\text { water }\end{array}$ & $\begin{array}{l}\text { Extract drunk } 250 \mathrm{ml} \\
\text { twice a day }\end{array}$ \\
\hline \multirow[t]{6}{*}{ Fabaceae } & $\begin{array}{l}\text { Piliostigma } \\
\text { thonningii } \\
\text { (Schumach.) } \\
\text { Milne-Redh. } \\
\text { AA-44-07 }\end{array}$ & $\begin{array}{l}\text { Open } \\
\text { grassland }\end{array}$ & T & L & STI & 2 & 6 & $\begin{array}{l}\text { Crushed, } \\
\text { mixed in cold } \\
\text { water }\end{array}$ & $\begin{array}{l}\text { Extract drunk } 750 \mathrm{ml} \\
\text { thrice a day }\end{array}$ \\
\hline & & & & St & Diarrhoea & & & $\begin{array}{l}\text { Crushed, } \\
\text { mixed in } \\
\text { warm water }\end{array}$ & $\begin{array}{l}\text { Drink one teaspoon a } \\
\text { day }\end{array}$ \\
\hline & $\begin{array}{l}\text { Cassia nigricans } \\
\text { Vahl. AA-31-07 }\end{array}$ & $\begin{array}{l}\text { Open } \\
\text { grassland }\end{array}$ & $\mathrm{SH}$ & St & Wound & 3 & 2 & Crushed & Apply on skin cuts \\
\hline & & & & $\mathrm{L}$ & Worms & & & $\begin{array}{l}\text { Crushed, } \\
\text { mixed in cold } \\
\text { water }\end{array}$ & Extract drunk \\
\hline & & & & L & Stomachache & & & Crushed & Smear on stomach \\
\hline & $\begin{array}{l}\text { Erythrina } \\
\text { abyssinica Lam. } \\
\text { AA-29-07 }\end{array}$ & Grassland & $\mathrm{T}$ & $\mathrm{R}$ & Toothache & 1 & 2 & $\begin{array}{l}\text { Crushed, } \\
\text { boiled }\end{array}$ & Massage tooth \\
\hline \multirow[t]{2}{*}{ Labiatae } & $\begin{array}{l}\text { Hoslundia } \\
\text { opposita Vahl. } \\
\text { AA-09-07 }\end{array}$ & $\begin{array}{l}\text { Open grass } \\
\text { land }\end{array}$ & $\mathrm{H}$ & R & Epilepsy & 2 & 6 & $\begin{array}{l}\text { Crushed, } \\
\text { mixed in cold } \\
\text { water }\end{array}$ & $\begin{array}{l}\text { Extract drunk two times a } \\
\text { day, applied as nasal } \\
\text { drop. }\end{array}$ \\
\hline & & & & $R$ & $\begin{array}{l}\text { Whole body } \\
\text { swelling }\end{array}$ & & & $\begin{array}{l}\text { Crushed, } \\
\text { boiled }\end{array}$ & Extract drunk \\
\hline \multirow[t]{5}{*}{ Lamiaceae } & $\begin{array}{l}\text { Clerodendrum } \\
\text { myricoides R.Br. \& } \\
\text { Vatke AA-30-07 }\end{array}$ & $\begin{array}{l}\text { Open } \\
\text { grassland }\end{array}$ & S & $\mathrm{R}$ & Body pains & 2 & 4 & Crushed & Rub on skin cuts \\
\hline & & & & $R$ & Cataracts & & & Crushed & $\begin{array}{l}\text { Extract dropped in eye } \\
\text { twice a day }\end{array}$ \\
\hline & $\begin{array}{l}\text { Ocimum } \\
\text { basilicum L. } \\
\text { AA-32-07 }\end{array}$ & $\begin{array}{l}\text { Compound } \\
\text { edge }\end{array}$ & $\mathrm{H}$ & L & Eye cataract & 3 & 3 & Crushed & $\begin{array}{l}\text { Extract squeezed, } \\
\text { dropped in eye twice a } \\
\text { day }\end{array}$ \\
\hline & & & & L & Fever & & & $\begin{array}{l}\text { Crushed, } \\
\text { mixed in } \\
\text { warm water }\end{array}$ & $\begin{array}{l}\text { Massage body, add in } \\
\text { bathing water }\end{array}$ \\
\hline & & & & $\mathrm{L}$ & Malaria & & & $\begin{array}{l}\text { Crushed, } \\
\text { mixed in } \\
\text { warm water }\end{array}$ & Extract drunk \\
\hline
\end{tabular}


Table 2 Medicinal plants their habits, growth habit, frequency of mention, plant part used, diseases treated, methods of preparation and administration (Continued)

\begin{tabular}{|c|c|c|c|c|c|c|c|c|}
\hline & $\begin{array}{l}\text { Vitex doniana } \\
\text { Sweet AA-25-07 }\end{array}$ & $\begin{array}{l}\text { Wooded } \\
\text { grassland }\end{array}$ & $\mathrm{T}$ & $\mathrm{R}$ & Eye disease & 1 & $\begin{array}{l}\text { Crushed, } \\
\text { mixed in cold } \\
\text { water }\end{array}$ & Extract dropped in eye \\
\hline \multirow[t]{8}{*}{ Leguminosae } & $\begin{array}{l}\text { Rhynchosia } \\
\text { densiflora Wall. } \\
\text { AA-27-07 }\end{array}$ & $\begin{array}{l}\text { Wooded } \\
\text { grassland }\end{array}$ & $\mathrm{SH}$ & $\mathrm{R}$ & Dysentery & 8 & $\begin{array}{l}\text { Crushed, } \\
\text { mixed in cold } \\
\text { water }\end{array}$ & $\begin{array}{l}\text { Extract drunk two } \\
\text { teaspoon twice a day }\end{array}$ \\
\hline & \multirow[t]{4}{*}{$\begin{array}{l}\text { Indigofera arrecta } \\
\text { Hochst.ex. A. Rich } \\
\text { AA-26-07 }\end{array}$} & \multirow[t]{4}{*}{$\begin{array}{l}\text { Open } \\
\text { garden }\end{array}$} & \multirow[t]{4}{*}{$\mathrm{SH}$} & $\mathrm{L}$ & $\begin{array}{l}\text { Body } \\
\text { swelling }\end{array}$ & 5 & Crushed & \multirow[b]{2}{*}{$\begin{array}{l}\text { Extract drunk } 200 \mathrm{ml} \\
\text { once a day }\end{array}$} \\
\hline & & & & $\mathrm{R}$ & $\begin{array}{l}\text { Round } \\
\text { worms }\end{array}$ & & $\begin{array}{l}\text { Crushed, } \\
\text { mixed in } \\
\text { warm water }\end{array}$ & \\
\hline & & & & $\mathrm{R}$ & Headache & & $\begin{array}{l}\text { Crushed, } \\
\text { mixed in cold } \\
\text { water }\end{array}$ & Extract drunk \\
\hline & & & & $\mathrm{R}$ & Sore throat & & $\begin{array}{l}\text { Crushed, } \\
\text { mixed in cold } \\
\text { water }\end{array}$ & Extract drunk twice a day \\
\hline & $\begin{array}{l}\text { Acacia hockii De } \\
\text { Wild AA-24-07 }\end{array}$ & $\begin{array}{l}\text { Open } \\
\text { grassland }\end{array}$ & $\mathrm{T}$ & $\mathrm{R}$ & $\begin{array}{l}\text { Malaria + } \\
\text { cough }\end{array}$ & 1 & $\begin{array}{l}\text { Crushed, } \\
\text { mixed in cold } \\
\text { water }\end{array}$ & $\begin{array}{l}\text { Extract drunk two times a } \\
\text { day }\end{array}$ \\
\hline & \multirow[t]{2}{*}{$\begin{array}{l}\text { Acacia sieberiana } \\
\text { Tausch AA-23-07 }\end{array}$} & \multirow[t]{2}{*}{$\begin{array}{l}\text { Wooded } \\
\text { grassland }\end{array}$} & \multirow[t]{2}{*}{$\mathrm{T}$} & $\mathrm{R}$ & Epilepsy & 1 & \multicolumn{2}{|c|}{$\begin{array}{l}\text { Crushed, Extract drunk } \\
\text { mixed in cold } \\
\text { water }\end{array}$} \\
\hline & & & & $\mathrm{R}$ & Dysentery & & $\begin{array}{l}\text { Crushed, } \\
\text { mixed in cold } \\
\text { water* }^{*}\end{array}$ & $\begin{array}{l}\text { Extract drunk half a } \\
\text { Aglass two times a day }\end{array}$ \\
\hline Loganiaceae & $\begin{array}{l}\text { Strychnos innocua } \\
\text { Delile. AA-12-07 }\end{array}$ & Swamps & $\mathrm{T}$ & $\mathrm{R}$ & Witchcraft & 1 & $\begin{array}{l}\text { Crushed, } \\
\text { mixed in cold } \\
\text { water }\end{array}$ & $\begin{array}{l}\text { Extract sprinkled on } \\
\text { patient }\end{array}$ \\
\hline \multirow[t]{10}{*}{ Meliaceae } & \multirow[t]{7}{*}{$\begin{array}{l}\text { Trichilia capensis } \\
\text { Pers. AA-22-07 }\end{array}$} & \multirow[t]{7}{*}{ Grassland, } & \multirow[t]{7}{*}{$\mathrm{SH}$} & $\mathrm{R}$ & Stomachache & 8 & $\begin{array}{l}\text { Crushed, } \\
\text { mixed in cold } \\
\text { water }\end{array}$ & $\begin{array}{l}\text { Extract drunk } 50 \mathrm{ml} \text { once } \\
\text { a day }\end{array}$ \\
\hline & & & & $\mathrm{R}$ & $\begin{array}{l}\text { Stops } \\
\text { miscarriage }\end{array}$ & & $\begin{array}{l}\text { Crushed, } \\
\text { mixed in cold } \\
\text { water }\end{array}$ & $\begin{array}{l}\text { Extract drunk half glass } \\
\text { twice a day }\end{array}$ \\
\hline & & & & $\mathrm{R}$ & West pain & & $\begin{array}{l}\text { Dried, } \\
\text { powdered }\end{array}$ & $\begin{array}{l}\text { Powder added in water } \\
\text { making } 10 \mathrm{ml} \text {, drunk } \\
\text { two times a day }\end{array}$ \\
\hline & & & & $\mathrm{R}$ & Urine pain & & $\begin{array}{l}\text { Crushed, } \\
\text { mixed in cold } \\
\text { water }\end{array}$ & $\begin{array}{l}\text { Extract drunk two times a } \\
\text { day }\end{array}$ \\
\hline & & & & $\mathrm{R}$ & $\begin{array}{l}\text { Back ache } \\
\text { after birth }\end{array}$ & & $\begin{array}{l}\text { Crushed, } \\
\text { mixed in cold } \\
\text { water }\end{array}$ & Extract drunk \\
\hline & & & & $\mathrm{R}$ & Worms & & $\begin{array}{l}\text { Crushed, } \\
\text { mixed in } \\
\text { water }\end{array}$ & Extract drunk \\
\hline & & & & $\mathrm{R}$ & $\begin{array}{l}\text { Diarrhoea, } \\
\text { cough }\end{array}$ & & $\begin{array}{l}\text { Crushed, } \\
\text { boiled }\end{array}$ & $\begin{array}{l}\text { Extract drunk } 200 \mathrm{ml} \\
\text { once a day }\end{array}$ \\
\hline & \multirow[t]{3}{*}{$\begin{array}{l}\text { Trichilia emetica } \\
\text { Vahl. AA-21-07 }\end{array}$} & \multirow[t]{3}{*}{$\begin{array}{l}\text { Open } \\
\text { grassland }\end{array}$} & \multirow[t]{3}{*}{$\mathrm{H}$} & $\mathrm{R}$ & Snake bite & \multirow[t]{3}{*}{11} & $\begin{array}{l}\text { Crushed, } \\
\text { mixed in cold } \\
\text { water }\end{array}$ & $\begin{array}{l}\text { Extract drunk, crushed } \\
\text { leaves rubbed on skin } \\
\text { cuts }\end{array}$ \\
\hline & & & & $\mathrm{R}$ & Stomachache & & $\begin{array}{l}\text { Crushed, } \\
\text { mixed in cold } \\
\text { water }\end{array}$ & Extract drunk once a day \\
\hline & & & & $\mathrm{R}$ & $\begin{array}{l}\text { prevent } \\
\text { poison }\end{array}$ & & $\begin{array}{l}\text { Crushed, } \\
\text { mixed in cold } \\
\text { water }\end{array}$ & Extract drunk \\
\hline
\end{tabular}


Table 2 Medicinal plants their habits, growth habit, frequency of mention, plant part used, diseases treated, methods of preparation and administration (Continued)

\begin{tabular}{|c|c|c|c|c|c|c|c|c|c|}
\hline Menispermaceae & $\begin{array}{l}\text { Cissampelos } \\
\text { mucronata A.Rich. } \\
\text { AA-33-07 }\end{array}$ & $\begin{array}{l}\text { Garden } \\
\text { edges }\end{array}$ & $\mathrm{H}$ & $R$ & $\begin{array}{l}\text { Abdominal } \\
\text { pain }\end{array}$ & 1 & 1 & $\begin{array}{l}\text { Crushed, } \\
\text { mixed in cold } \\
\text { water }\end{array}$ & $\begin{array}{l}\text { Extract drunk three times } \\
\text { a day }\end{array}$ \\
\hline Mimosaceae & $\begin{array}{l}\text { Albizia coriaria } \\
\text { Welw. AA-58-07 }\end{array}$ & $\begin{array}{l}\text { Wooded } \\
\text { grassland }\end{array}$ & $T$ & B & Diarrhoea & 1 & 1 & $\begin{array}{l}\text { Crushed, } \\
\text { mixed in cold } \\
\text { water }\end{array}$ & Extract drunk \\
\hline \multirow[t]{2}{*}{ Moraceae } & $\begin{array}{l}\text { Ficus vallis } \\
\text { Chaude AA-20-07 }\end{array}$ & $\begin{array}{l}\text { Wooded } \\
\text { grassland }\end{array}$ & $T$ & $R$ & $\begin{array}{l}\text { Dysentery, } \\
\text { diarrhea }\end{array}$ & 3 & 7 & $\begin{array}{l}\text { Crushed, } \\
\text { mixed in cold } \\
\text { water }\end{array}$ & $\begin{array}{l}\text { Extract drunk half glass } \\
\text { once a day }\end{array}$ \\
\hline & & & & B & Ring worm & & & Sap collected & $\begin{array}{l}\text { Smeared on affected area } \\
\text { twice a day }\end{array}$ \\
\hline \multirow[t]{3}{*}{ Musaceae } & $\begin{array}{l}\text { Musa spp } \\
\text { AA-69-07 }\end{array}$ & Garden & $T$ & $\mathrm{~F}$ & Diarrhoea & 3 & 1 & Sap collected & Sap drunk thrice a day \\
\hline & & & & $\mathrm{Fl}$ & Wound & & & $\begin{array}{l}\text { Crushed, } \\
\text { mixed in cold } \\
\text { water }\end{array}$ & Extract applied on wound \\
\hline & & & & B & Ring worm & & & Crushed & $\begin{array}{l}\text { Smear on affected area } \\
\text { once a day }\end{array}$ \\
\hline Myrtaceae & $\begin{array}{l}\text { Eucalyptus } \\
\text { globulus Labill. } \\
\text { AA-68-07 }\end{array}$ & Home stead & $T$ & $L$ & Cough & 1 & 5 & $\begin{array}{l}\text { Crushed, } \\
\text { boiled }\end{array}$ & $\begin{array}{l}\text { Extract drunk four } \\
\text { teaspoon twice a day }\end{array}$ \\
\hline \multirow[t]{2}{*}{ Papilionaceae } & $\begin{array}{l}\text { Crotalaria } \\
\text { ochroleuca } \\
\text { G.Don AA-04-07 }\end{array}$ & Garden & $\mathrm{SH}$ & L & Stomachache & 1 & 1 & $\begin{array}{l}\text { Crushed, } \\
\text { boiled }\end{array}$ & Eaten \\
\hline & $\begin{array}{l}\text { Cajanus cajan (L.) } \\
\text { Druce AA-17-07 }\end{array}$ & Garden & $\mathrm{SH}$ & L & Malaria & 1 & 1 & $\begin{array}{l}\text { Crushed, } \\
\text { mixed in cold } \\
\text { water }\end{array}$ & $\begin{array}{l}\text { Extract drunk } 100 \mathrm{ml} \\
\text { once a day }\end{array}$ \\
\hline \multirow[t]{3}{*}{ Poaceae } & $\begin{array}{l}\text { Imperata cylindra } \\
\text { P.Beauv. } \\
\text { AA-67-07 }\end{array}$ & $\begin{array}{l}\text { Open } \\
\text { grassland }\end{array}$ & G & $\mathrm{R}$ & $\begin{array}{l}\text { Abdominal } \\
\text { pain }\end{array}$ & 1 & 1 & $\begin{array}{l}\text { Crushed, } \\
\text { mixed in cold } \\
\text { water }\end{array}$ & Extract drunk \\
\hline & $\begin{array}{l}\text { Pennisetum } \\
\text { trachyphyllum } \\
\text { Pilg. AA-66-07 }\end{array}$ & $\begin{array}{l}\text { Garden, dry } \\
\text { land }\end{array}$ & G & $R$ & $\begin{array}{l}\text { Abdominal } \\
\text { pain }\end{array}$ & 1 & 1 & $\begin{array}{l}\text { Crushed, } \\
\text { mixed in cold } \\
\text { water }\end{array}$ & Extract drunk \\
\hline & $\begin{array}{l}\text { Sporobulus } \\
\text { africanus (Poir.) } \\
\text { Roebyns } \\
\text { AA-65-07 }\end{array}$ & $\begin{array}{l}\text { Open } \\
\text { grassland }\end{array}$ & G & $R$ & $\begin{array}{l}\text { Retained } \\
\text { placenta }\end{array}$ & 1 & 2 & $\begin{array}{l}\text { Crushed, } \\
\text { mixed in cold } \\
\text { water }\end{array}$ & $\begin{array}{l}\text { Extract drunk500 } \mathrm{ml} \text { once } \\
\text { a day }\end{array}$ \\
\hline \multirow[t]{4}{*}{ Polygolaceae } & $\begin{array}{l}\text { Securidaca } \\
\text { longipedunculata } \\
\text { Fres. AA-19-07 }\end{array}$ & $\begin{array}{l}\text { Open } \\
\text { grassland }\end{array}$ & T & $\mathrm{R}$ & Body pains, & 4 & 19 & Crushed & $\begin{array}{l}\text { Rubbed on skin cuts } \\
\text { once a day }\end{array}$ \\
\hline & & & & $\mathrm{R}$ & Headache & & & Crushed & $\begin{array}{l}\text { Rubbed on skin cuts } \\
\text { once a day }\end{array}$ \\
\hline & & & & $\mathrm{R}$ & Skin disease & & & $\begin{array}{l}\text { Crushed, } \\
\text { mixed in cold } \\
\text { water }\end{array}$ & $\begin{array}{l}\text { Rubbed on affected area } \\
\text { three times a day }\end{array}$ \\
\hline & & & & $R$ & $\begin{array}{l}\text { Body ache } \\
\text { due to } \\
\text { witchcraft }\end{array}$ & & & $\begin{array}{l}\text { Crushed, } \\
\text { mixed in cold } \\
\text { water }\end{array}$ & $\begin{array}{l}\text { Rubbed on skin cuts } \\
\text { once a day }\end{array}$ \\
\hline \multirow[t]{4}{*}{ Ranunculaceae } & $\begin{array}{l}\text { Clematis hirusta } \\
\text { Guill. \& Perr. } \\
\text { AA-05-07 }\end{array}$ & $\begin{array}{l}\text { Anthill on } \\
\text { Open } \\
\text { grassland }\end{array}$ & $\mathrm{H}$ & $R$ & Swelling & 4 & 14 & Crushed & Massage affected area \\
\hline & & & & $\mathrm{R}$ & STI & & & $\begin{array}{l}\text { Crushed, } \\
\text { mixed in cold } \\
\text { water }\end{array}$ & $\begin{array}{l}\text { Extract drunk two glass } \\
\text { thrice a day }\end{array}$ \\
\hline & & & & $R$ & Cough & & & $\begin{array}{l}\text { Crushed, } \\
\text { boiled }\end{array}$ & Extract drunk twice a day \\
\hline & & & & $\mathrm{Fl}$ & Flu & & & Crushed & Inhaled \\
\hline
\end{tabular}


Table 2 Medicinal plants their habits, growth habit, frequency of mention, plant part used, diseases treated, methods of preparation and administration (Continued)

\begin{tabular}{|c|c|c|c|c|c|c|c|c|c|}
\hline \multirow[t]{7}{*}{ Rubiaceae } & \multirow[t]{6}{*}{$\begin{array}{l}\text { Sarcocephalus } \\
\text { latifolius (SM.) E.A. } \\
\text { Bruce AA-51-07 }\end{array}$} & \multirow[t]{6}{*}{ Grassland } & \multirow[t]{6}{*}{$\mathrm{SH}$} & $\mathrm{R}$ & \multirow{2}{*}{$\begin{array}{l}\text { Piles } \\
\text { Scrotal } \\
\text { hernia }\end{array}$} & \multirow[t]{2}{*}{8} & \multirow[t]{6}{*}{12} & \multirow{2}{*}{$\begin{array}{l}\text { Burnt } \\
\text { together with } \\
\text { millet husk } \\
\text { Crushed, } \\
\text { mixed in cold } \\
\text { water }\end{array}$} & \multirow{2}{*}{$\begin{array}{l}\text { Direct smoke to anus } \\
\text { Extract drunk } 20 \mathrm{ml} \text { once } \\
\text { a day for a month }\end{array}$} \\
\hline & & & & $\mathrm{R}$ & & & & & \\
\hline & & & & $R$ & $\begin{array}{l}\text { Cough, } \\
\text { stomachache }\end{array}$ & & & $\begin{array}{l}\text { Crushed, } \\
\text { boiled }\end{array}$ & $\begin{array}{l}\text { Extract drunk } 200 \mathrm{ml} \\
\text { once a day }\end{array}$ \\
\hline & & & & R & STDs, worms & & & $\begin{array}{l}\text { Crushed, } \\
\text { boiled }\end{array}$ & $\begin{array}{l}\text { Extract drunk one glass } \\
\text { twice a day }\end{array}$ \\
\hline & & & & R & Diarrhoea & & & $\begin{array}{l}\text { Crushed, } \\
\text { mixed in cold } \\
\text { water }\end{array}$ & $\begin{array}{l}\text { Extract drunk half glass } \\
\text { thrice a day }\end{array}$ \\
\hline & & & & $\mathrm{R}$ & Dysentery & & & $\begin{array}{l}\text { Crushed, } \\
\text { mixed in cold } \\
\text { water }\end{array}$ & $\begin{array}{l}\text { Extract drunk } 200 \mathrm{ml} \\
\text { thrice a day }\end{array}$ \\
\hline & $\begin{array}{l}\text { Vangueria } \\
\text { apiculata K. } \\
\text { Schum AA-16-07 }\end{array}$ & Forest edge & & S & $\begin{array}{l}\text { Swollen feet } \\
\text {, body }\end{array}$ & 1 & 1 & $\begin{array}{l}\text { Crushed, } \\
\text { mixed in cold } \\
\text { water }\end{array}$ & $\begin{array}{l}\text { Extract drunk half glass } \\
\text { three times a day }\end{array}$ \\
\hline Sapotaceae & $\begin{array}{l}\text { Vitallaria } \\
\text { paradoxum } \\
\text { (C.F. Gaertn) } \\
\text { Hepper AA-14-07 }\end{array}$ & $\begin{array}{l}\text { Wooded } \\
\text { grassland }\end{array}$ & T & B & Diarrhoea & 1 & 3 & $\begin{array}{l}\text { Dried, } \\
\text { powder } \\
\text { mixed in } \\
\text { water }\end{array}$ & $\begin{array}{l}\text { Drunk } 20 \mathrm{ml} \text { two times a } \\
\text { day }\end{array}$ \\
\hline \multirow[t]{2}{*}{ Simaroubaceae } & $\begin{array}{l}\text { Harrisonia } \\
\text { occidentalist (Eng) } \\
\text { L.AA-15-07 }\end{array}$ & Ant hills & $\mathrm{SH}$ & $\mathrm{R}$ & Worms & 2 & 2 & $\begin{array}{l}\text { Crushed, } \\
\text { mixed in } \\
\text { warm water } \\
\end{array}$ & $\begin{array}{l}\text { Extract drunk } 500 \mathrm{ml} \mathrm{a} \\
\text { day }\end{array}$ \\
\hline & & & & $L$ & $\begin{array}{l}\text { Sores on } \\
\text { head of } \\
\text { children }\end{array}$ & & & Crushed & $\begin{array}{l}\text { Rubbed on affected area } \\
\text { twice a day }\end{array}$ \\
\hline \multirow[t]{10}{*}{ Solanaceae } & $\begin{array}{l}\text { Capsicum } \\
\text { frutescens Rodsch. } \\
\text { AA-13-07 }\end{array}$ & $\begin{array}{l}\text { Under big } \\
\text { trees }\end{array}$ & $\mathrm{SH}$ & S & Backache & 1 & 1 & Crushed & $\begin{array}{l}\text { Crushed bark rubbed on } \\
\text { skin cuts }\end{array}$ \\
\hline & $\begin{array}{l}\text { Solanum sp } \\
\text { AA-10-07 }\end{array}$ & $\begin{array}{l}\text { Ant hills, } \\
\text { open } \\
\text { grassland }\end{array}$ & $\mathrm{SH}$ & $\mathrm{R}$ & STI & 5 & 2 & $\begin{array}{l}\text { Crushed, } \\
\text { mixed in cold } \\
\text { water }\end{array}$ & Extract drunk \\
\hline & & & & R & Ear disease & & & $\begin{array}{l}\text { Crushed, } \\
\text { mixed in cold } \\
\text { water }\end{array}$ & $\begin{array}{l}\text { Extract dropped in ear } \\
\text { thrice a day }\end{array}$ \\
\hline & & & & $\mathrm{R}$ & Epilepsy & & & $\begin{array}{l}\text { Crushed, } \\
\text { mixed in cold } \\
\text { water }\end{array}$ & \\
\hline & & & & $\mathrm{R}$ & Diarrhoea & & & $\begin{array}{l}\text { Crushed, } \\
\text { mixed in cold } \\
\text { water }\end{array}$ & $\begin{array}{l}\text { Extract drunk two } \\
\text { teaspoon twice a day }\end{array}$ \\
\hline & & & & $\mathrm{R}$ & Headache & & & $\begin{array}{l}\text { Crushed, } \\
\text { mixed in cold } \\
\text { water }\end{array}$ & Extract drunk \\
\hline & $\begin{array}{l}\text { Solanum } \\
\text { aculeatissimum } \\
\text { Jacq AA-28-07 }\end{array}$ & Homestead & $\mathrm{SH}$ & R & Witchcraft & 5 & 5 & Crushed & Rub on skin cuts \\
\hline & & & & R & Hydrocelle & & & $\begin{array}{l}\text { Crushed, } \\
\text { mixed in cold } \\
\text { water }\end{array}$ & Extract drunk \\
\hline & & & & $\mathrm{R} / \mathrm{F}$ & snake bite & & & $\begin{array}{l}\text { Crushed, } \\
\text { mixed in cold } \\
\text { water }\end{array}$ & Extract drunk \\
\hline & & & & $\mathrm{F}$ & $\begin{array}{l}\text { Bone, } \\
\text { muscle } \\
\text { inflammation }\end{array}$ & & & $\begin{array}{l}\text { Crushed, } \\
\text { mixed in cold } \\
\text { water* }\end{array}$ & Extract drunk \\
\hline
\end{tabular}


Table 2 Medicinal plants their habits, growth habit, frequency of mention, plant part used, diseases treated, methods of preparation and administration (Continued)

\begin{tabular}{|c|c|c|c|c|c|c|c|c|c|}
\hline Tiliaceae & $\begin{array}{l}\text { Grewia mollis } \\
\text { Juss. AA-70-07 }\end{array}$ & $\begin{array}{l}\text { Open } \\
\text { grassland }\end{array}$ & $\mathrm{T}$ & R & $\begin{array}{l}\text { Swollen } \\
\text { body part }\end{array}$ & 1 & 1 & Scraped & Plastered on swelling \\
\hline Tricholomataceae & $\begin{array}{l}\text { Termitomyces } \\
\text { microcarpus } \\
\text { AA-71-07 }\end{array}$ & Forest & & R & Boils & 1 & 1 & Crushed & Smeared on affected area \\
\hline \multirow[t]{2}{*}{ Umbellifereae } & $\begin{array}{l}\text { Steganofaenia } \\
\text { oraliacea } \\
\text { AA-63-07 }\end{array}$ & $\begin{array}{l}\text { Open } \\
\text { grassland }\end{array}$ & $\mathrm{SH}$ & R & Measles & 2 & 2 & Crushed & Rubbed all over skin \\
\hline & & & & R & $\begin{array}{l}\text { Swollen } \\
\text { body }\end{array}$ & & & $\begin{array}{l}\text { Dried, } \\
\text { powdered }\end{array}$ & $\begin{array}{l}\text { Added in one glass of } \\
\text { water, drunk twice a day }\end{array}$ \\
\hline \multirow[t]{4}{*}{ Urticaceae } & $\begin{array}{l}\text { Urtica massaica } \\
\text { Mildbr. AA-08-07 }\end{array}$ & $\begin{array}{l}\text { Forest, } \\
\text { swamp }\end{array}$ & $\mathrm{SH}$ & L & Headache & 4 & 4 & Crushed & Rubbed on forehead \\
\hline & & & & $R$ & $\begin{array}{l}\text { Menstrual } \\
\text { pain }\end{array}$ & & & Crushed & $\begin{array}{l}\text { Extract drunk four } \\
\text { teaspoon twice a day }\end{array}$ \\
\hline & & & & R & Boils & & & Crushed & $\begin{array}{l}\text { Extract smeared on } \\
\text { affected area once }\end{array}$ \\
\hline & & & & R & Cough & & & $\begin{array}{l}\text { Crushed, } \\
\text { mixed in cold } \\
\text { water }\end{array}$ & Extract drunk \\
\hline \multirow[t]{7}{*}{ Verbenaceae } & $\begin{array}{l}\text { Lanatana camara } \\
\text { L. AA-03-07 }\end{array}$ & $\begin{array}{l}\text { Garden } \\
\text { edge, } \\
\text { roadside }\end{array}$ & $\mathrm{SH}$ & L & Ringworms & 4 & 5 & $\begin{array}{l}\text { Dried, } \\
\text { powdered }\end{array}$ & Smeared on affected area \\
\hline & & & & L & Cataracts & & & $\begin{array}{l}\text { Crushed, } \\
\text { mixed in cold } \\
\text { water }\end{array}$ & Extract dropped in eye \\
\hline & & & & R & snake bite & & & $\begin{array}{l}\text { Crushed, } \\
\text { mixed in cold } \\
\text { water }\end{array}$ & Extract drunk $250 \mathrm{ml}$ \\
\hline & & & & R & Epilepsy & & & $\begin{array}{l}\text { Crushed, } \\
\text { mixed in cold } \\
\text { water }\end{array}$ & Extract drunk \\
\hline & $\begin{array}{l}\text { Clerodendrum } \\
\text { umbellatum Poir } \\
\text { AA-06-07 }\end{array}$ & Gardens & $\mathrm{SH}$ & $\mathrm{R}$ & Cough & 3 & 28 & $\begin{array}{l}\text { Crushed, } \\
\text { boiled }\end{array}$ & Extract drunk third a glass \\
\hline & & & & L & Poison & & & $\begin{array}{l}\text { Crushed, } \\
\text { mixed in cold } \\
\text { water }\end{array}$ & Extract drunk \\
\hline & & & & $\mathrm{L}$ & $\begin{array}{l}\text { Abdominal } \\
\text { pain }\end{array}$ & & & $\begin{array}{l}\text { picked, } \\
\text { cleaned }\end{array}$ & Chewed \\
\hline \multirow[t]{4}{*}{ Vitaceae } & $\begin{array}{l}\text { Cyphostemma } \\
\text { adenocaule } \\
\text { Descoings. } \\
\text { ex Wild \& R.B. } \\
\text { Drumm. } \\
\text { AA-01-07 }\end{array}$ & $\begin{array}{l}\text { Open } \\
\text { grassland }\end{array}$ & C & R & Wounds & 4 & 3 & Sap collected & Rubbed on skin cuts \\
\hline & & & & $R$ & Abortion & & & $\begin{array}{l}\text { Crushed, } \\
\text { mixed in cold } \\
\text { water }\end{array}$ & $\begin{array}{l}\text { Extract drunk three } \\
\text { teaspoon three times a } \\
\text { day }\end{array}$ \\
\hline & & & & $R$ & Boils & & & Crushed & $\begin{array}{l}\text { Extract smeared on } \\
\text { affected area once }\end{array}$ \\
\hline & & & & $R$ & Cough & & & $\begin{array}{l}\text { Crushed, } \\
\text { mixed in cold } \\
\text { water }\end{array}$ & Extract drunk \\
\hline \multirow[t]{2}{*}{ Zingiberaceae } & $\begin{array}{l}\text { Zingiber officinale } \\
\text { Roscoe AA-34-07 }\end{array}$ & Homestead & $\mathrm{H}$ & R & Meningitis & 2 & 4 & Crushed & $\begin{array}{l}\text { Rubbed on skin cuts } \\
\text { once }\end{array}$ \\
\hline & & & & $R$ & Cough & & & $\begin{array}{l}\text { Crushed, } \\
\text { warm water } \\
\text { added }\end{array}$ & Drunk \\
\hline
\end{tabular}


Table 2 Medicinal plants their habits, growth habit, frequency of mention, plant part used, diseases treated, methods of preparation and administration (Continued)

\begin{tabular}{|c|c|c|c|c|c|c|c|}
\hline \multirow[t]{2}{*}{$\begin{array}{l}\text { Aframomum } \\
\text { angustifolium K. } \\
\text { Schum AA-39-07 }\end{array}$} & $\begin{array}{l}\text { Open } \\
\text { wooded } \\
\text { grassland }\end{array}$ & S & Cholera & 2 & 11 & $\begin{array}{l}\text { Crushed, } \\
\text { mixed in cold } \\
\text { water }\end{array}$ & Drunk \\
\hline & & S & Diarrhoea & & & $\begin{array}{l}\text { Crushed, } \\
\text { mixed in } \\
\text { warm water }\end{array}$ & $\begin{array}{l}\text { Drunk } 50 \mathrm{ml} \text { two } \\
\text { times a day for four days }\end{array}$ \\
\hline
\end{tabular}

Key: Plant Habit: SH-Shrub T-Tree C- Climber H-Herb G-Grass.

Plant part used: R-Roots L-Leaves B-Bark S-Seeds F-Fruit S-Stem F-Flower.

Mode of preparation: $(1 * 2 * 3 *)$ - Used in combination with other plant species.

$4 x$ - Mixed in oil.

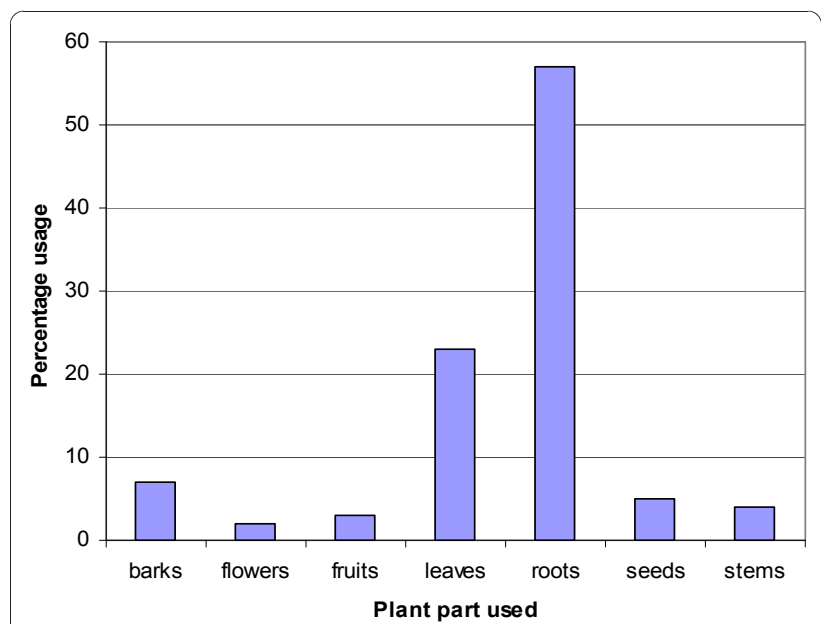

Figure 2 Different plant parts used for medicinal purpose and their percentages.

Table 3 Source of knowledge on medicinal plant species among the people of Ngai and Otwal sub counties in Oyam District

\begin{tabular}{ccc}
\hline Source of information & Frequency & Percentage \\
\hline Parents & 42 & 40 \\
Peers & 5 & 4.8 \\
Grandparents & 37 & 35.6 \\
Traditional healers & 13 & 13 \\
In laws & 3 & 2.9 \\
Dreams & 4 & 3.8 \\
Total & $\mathbf{1 0 4}$ & $\mathbf{1 0 0 . 1}$ \\
\hline
\end{tabular}

Table 4 Showing why medicinal plant species are in use

\begin{tabular}{lll}
\hline Reason for use & Frequency & $\%$ \\
\hline Medical facilities far & 35 & 23 \\
Poverty & 35 & 23 \\
Conventional medicine don't work & 7 & 5 \\
Medicinal plant species effective & 51 & 34 \\
Hospitals lack medicines & 7 & 5 \\
Advice from medical workers & 4 & 3 \\
Easy to access & 12 & 8 \\
\hline
\end{tabular}

some of the plant species are hard to find and one has to risk going to restricted conservation areas to get the plant species. Since the knowledge comes at a price, many people are even too poor to pay for the herbalist services, hence a reduction in number of clients.

The disappearance of medicinal plant species can also be attributed to over use, agricultural activities and insecurity. Domestication of medicinal plant species is probably not taken seriously. Some medicinal plant species which have been proved potent have been over used $[4,20]$. The mode of harvesting which involves the use of roots also posed a threat to the existence of these plant species. In most of the plant species, their roots were being used.

\section{Conclusions}

The 71 medicinal plant species of cultivated and wild types were greatly utilized for treating a total of 41 different ailments by people of Ngai and Otwal Sub Counties. Thirty nine percent of the recorded plant species were reported for treating stomach related ailments. The most commonly mentioned plant species by respondents were Clerodendrum umbellatum Poir (25\%). Of the total of plant species documented, $25 \%$ were edible and formed part of local diet. The main sources of indigenous knowledge of medicinal plant species were parents at $40 \%$.

Roots were the most commonly harvested plant part of the medicinal plant species compared to any other part. The most common method of preparation of medicinal plant species before being administered was found to be applied to most plant species. However, it was noted that some of these medicinal plant species are disappearing very first. The disappearance of medicinal plant species can be attributed to over use, agricultural activities and insecurity. Domestication of medicinal plant species is probably not taken seriously.

The use of medicinal plant species in primary health care is still a common practice in Ngai and Otwal Sub-County. The inadequate health services and abject poverty still make these people dependent on herbal medicine for their day to day health needs. 
The generation gap caused by the over 20 years of insurgency in the area has brought about knowledge gap between the young and the old with regard to medicinal plant species.

\section{Recommendations}

- There is need for ex-situ conservation of the useful medicinal plant species

- There is need for community awareness and education concerning the values of medicinal plant species of the area especially among the young people.

- Further studies should be done on the medicinal plant species to determine their pharmacological potentials.

- Government should develop policy to integrate use of medicinal plant species in health care at national level

\begin{abstract}
Acknowledgements
Our most sincere gratitude to the sponsor, NORAD Medicinal plant species Project through the Department of Botany Makerere University, The Staff of Ngai Health Center III, Field assistant, leaders of Lango Cultural Center, local leaders, traditional healers, traditional birth attendants, the resource users and all respondents, in Ngai and Otwal Sub counties in Oyam Districts who provided the information.
\end{abstract}

\section{Author details}

'Department of Botany, Makerere University, P.O Box 7062, Kampala, Uganda. ${ }^{2}$ Institute of Environment and Natural Resources Makerere University, P.O Box 7062, Kampala, Uganda.

\section{Authors' contributions}

AA identified the research area and title, collected field data, carried out statistical analysis and drafted the manuscript. MMK and $\mathrm{OOH}$ participated in refining the title, formulation of the research problem, data analysis and drafting as well as enrichment of the manuscript. All authors read and approved the final manuscript.

\section{Competing interests}

The authors declare that they have no competing interests.

Received: 17 July 2010 Accepted: 17 January 2011

Published: 17 January 2011

\section{References}

1. WHO: Mental health Global Action Program (mhGAP) World Health Organization, Geneva, Switzerland; 2002

2. Tabuti Robert SR: Locally used plant species in Bulamogi County, Uganda diversity, modes of utilisation. PhD thesis Agricultural University of Norway; 2003.

3. Kamatenesi MM, Makawiti DW, Oryem-Origa H, Olwa-Odyek : Enthnopharmacological screenings of Vernonia amygdalina and Cleome gynandra traditionally used in child birth in Western Uganda.Edited by: Midwo JO, Yenesew Abiy, Derese Solomon 2005, 81-89, the proceeding of the 11th NAPRECA Symposium on natural products and drug discovery: 912 Antananarivo.

4. World Health Organization: Traditional Medicines Strategy 2002-2005. Geneva; 2002.

5. Katende AB, Ssegwa P, Birnie A: Wild food plant species and Mushrooms of Uganda 1999, Regional Land Management Unit (RELMA), SIDA, Technical Handbook No 19, Nairobi.

6. Kamatenesi MM, Oryem-Origa H: Medicinal plant species used to induce labour during childbirth in western Uganda. Journal of Ethnopharmacology 2006, 109:1-9.
7. Katuura E, Waako P, Ogwal-Okeng J, Bukenya-Ziraba R: Traditional treatment of malaria in Mbarara District, western Uganda. African Journal of Ecology 2007, 45(Suppl. 1):48-51.

8. Rukia Sanogo: Analgesic and anti-inflamatory activities of the aqueous extracts of Maytenus senegalensis, Sterospermum kunthianum and Trichilia emetica used in the treatment of dysmenorrhoea in Mali (abstract). The 12th NAPRECA Symposium 2007, 40.

9. Tsey Komla: Traditional medicine in contemporary Ghana: A public policy analysis. Social Science \& Medicine 1997, 45:1065-1074.

10. Elujoba A, Odeleye M, Ogunyemi CM: Traditional medicine development for medical and Dental primary health care delivery system in Africa. Afr J Traditional, Complementary and Alternative Medicines 2005, 2(1):46-61.

11. Ibe $A E$, Nwufo Martin I: Identification, Collection and Domestication of Medicinal Plant species in Southeastern Nigeria. Africa Development 2005, 30:66-77.

12. Oyam District Achievements One Year of Development - June 2006 May 2007. [http://www.enteruganda.com/brochures/oyam05.htm].

13. Oyam District. [http://en.wikipedia.org/wiki/Oyam_District].

14. Martins GJ: Ethnobotany: A methods Manual London: Chapman and Hall; 1995.

15. Oryem-origa $H$, Katende $A B$, Kakudidi EKZ: Enthobotanical studies of Mabira forest area, central Uganda. African Academy of Science Special Edition 2002, 169-181.

16. Akerreta Silvia, Cavero Yolanda Rita, Calvo Isabel María: First comprehensive contribution to medical ethnobotany of Western Pyrenees. Journal of Ethnobiology and Ethnomedicine 2007, 3:26.

17. Okello J, Ssegawa P: Medicinal plant species used by communities of Ngai Sub county, Apac District, northern Uganda. African Journal of Ecology 2007, 45:76-83.

18. Bhattarai Shandesh, Chaudhary PRam, Quave LCassandra, Taylor SLRobin: The use of medicinal plant species in the trans-himalayan arid zone of Mustang district, Nepal. Journal of Ethnobiology and Ethnomedicine 2010, 6:14.

19. Waako PJ, Katuura E, smith P, Folb P: East Africa medicinal plant species as a source of lead compounds for development of new antimalarial drugs. African Journal of Ecology 2007, 45(Suppl. 1):102-106, 5.

20. Alexis Okeowo: "Sex tree", other medicinal plant species near extinction in Uganda. National Geographic News 2007.

doi:10.1186/1746-4269-7-7

Cite this article as: Kamatenesi et al:: Medicinal plants of Otwal and Ngai Sub Counties in Oyam District, Northern Uganda. Journal of Ethnobiology and Ethnomedicine 2011 7:7.

\section{Submit your next manuscript to BioMed Central and take full advantage of:}

- Convenient online submission

- Thorough peer review

- No space constraints or color figure charges

- Immediate publication on acceptance

- Inclusion in PubMed, CAS, Scopus and Google Scholar

- Research which is freely available for redistribution 